\title{
Determination of spallation neutron flux through spectral adjustment techniques
}

\author{
M.A. Mosby ${ }^{1,}$, J.W. Engle ${ }^{1,}$, K.R. Jackman ${ }^{1}$, F.M. Nortier ${ }^{1}$, E.R. Birnbaum ${ }^{1}$, \\ Los Alamos National Laboratory, P.O. Box 1663, Los Alamos, NM 87545, USA
}

\begin{abstract}
The Los Alamos Isotope Production Facility (IPF) creates medical isotopes using a proton beam impinged on a target stack. Spallation neutrons are created in the interaction of the beam with target. The use of these spallation neutrons to produce additional radionuclides has been proposed. However, the energy distribution and magnitude of the flux is not well understood. A modified SAND-II spectral adjustment routine has been used with radioactivation foils to determine the differential neutron fluence for these spallation neutrons during a standard IPF production run.
\end{abstract}

Keywords: Spallation neutrons; isotope production; proton irradiation; spectral unfolding

\section{Introduction}

The Isotope Production Facility (IPF) produces isotopes both for medicine and research using a $100 \mathrm{MeV}$ proton beam generated by the Los Alamos Neutron Science 5 Center (LANSCE). The proton beam is impinged on a target stack whose multiple targets subtend different energy regions of the incident proton beam. In practice, the high demand for certain routinely produced isotopes results in oversubscription of the primary proton beam time.

The interaction of the proton beam with the target ${ }_{45}$ stack is known to also generate secondary spallation neutrons. Use of these spallation neutrons has been proposed to symbiotically generate nuclei of medical and scientific interest, especially those that cannot be produced by the

15 IPF primary proton beam or in reactors where the neutron energy distribution is distinctly different and dominated by thermal neutrons. The secondary neutron flux created at IPF is of particular interest to create isotopes not feasible through other methods.

Proton bombardment is useful for creating neutrondeficient nuclei, generally positron emitters. Low-energy neutrons can be used for capture reactions, which produce nuclei one neutron richer than the target. Neutronrich nuclei further from stability can only be produced 25 through high energy neutron reactions, i.e. (n,p), (n,pn), $(\mathrm{n}, \alpha),(\mathrm{n}, 2 \mathrm{n}),(\mathrm{n}, 3 \mathrm{n})$, etc. Additionally, high energy neutrons induce nuclear transmutation, forming radionuclides absent added carrier mass. These small masses of radioac- ${ }^{6}$ tive product are nevertheless highly detectable thanks to their radioactive emissions, enabling study of biological processes without perturbing the physiological system or treatment of disease that is highly specific to receptor systems with very low in vivo density. Radionuclides of in- ${ }^{65}$ terest to produce using high energy neutrons include ${ }^{67} \mathrm{Cu}$

Email address: mosbym@lanl.gov (M.A. Mosby) in quantity by the medical community.

For the purposes of yield and purity predictions of the residual nuclei created, the IPF secondary neutron flux has been modeled in MCNPX but has not been directly verified experimentally due to spatial challenges imposed by the production facility configuration. Direct measurement of the neutron flux via time-of-flight is not feasible as the target chamber for IPF is located 12 meters below a hot cell in a compact, heavily shielded water-filled irradiation chamber.

The measurement of neutron fluxes in reactors, which often present similar spatial challenges, has been accomplished using radioactivation foils as an indirect probe. Spectral adjustment techniques are then used to adjust predictions of the flux using quantified radioactive residuals produced in these foils. A similar approach is presented here, using the radioactivation of selected foils to probe the secondary neutron flux produced at IPF. The results are compared with calculated activities from an MCNPX simulation, and the calculated neutron flux is adjusted using a modified SAND-II code in order to maximize agreement with the experimental measurements.

\section{Material and methods}

This experiment and resulting activity measurements are described in detail in [1]. In summary, high-purity metal reaction foils were placed downstream of the traditional foil stack used in IPF production irradiations. A schematic of the standard target stack used in the production of ${ }^{82} \mathrm{Sr}$ and ${ }^{68} \mathrm{Ge}$ is shown in Fig 1. This stack consists of two targets of Inconel-encapsulated rubidium chloride and a third of niobium-encapsulated liquid gallium metal, in which the proton beam is stopped. Seven foils, punched into discs with diameter $10.32 \pm 0.05 \mathrm{~cm}$ to 
match calibrated geometries for later analysis, were used vation foils: Al, Co, Y, Au, Bi, Ni, Zn. The activation foils were mounted directly downstream of the standard RbCl-RbCl-Ga IPF target stack, placing the foils in 120 a neutron flux consistent with IPF production conditions. Radioactivation foil samples were enclosed in two single 75 layers of $25 \mu \mathrm{m}$ thick Kapton tape to facilitate trapping of gaseous, radioactive products and to isolate samples from one another during irradiation.

The irradiation foil stack was exposed to standard IPF production conditions. The target stack received a $200 \mu \mathrm{A}$

80 proton current for four minutes to thermally condition upstream $\mathrm{RbCl}$ targets followed by a $230 \mu \mathrm{A}$ proton current for one hour.

Following irradiation, the foils were removed and transported to the LANL Nuclear and Radiochemistry group 85 counting room for $\gamma$-spectrometry using a high-purity germanium (HPGe) ORTEC GEM p-type aluminum-windowed detector. Details of the counting procedure and subse-135 quent analysis have been reported previously in 2 and [3]. The photopeaks from the $\gamma$-spectra were analyzed us90 ing an in-house code, SPECANAL, using gamma energies and intensities from the National Nuclear Data Center's (NNDC's) database. The activity at end of irradiation ${ }_{140}$ was determined from an exponential fit to the measured decay curve.

\subsection{Modifications of adjustment procedure}

The SAND-II [4] code is a statistical adjustment pro-145 cedure that iteratively modifies the neutron flux based on measured activation foil yields and calculated yields determined by using the input flux and known cross sections, where the input flux's characteristics are generally obtained from a simulation such as MCNP. The activity ${ }^{150}$ (a) in each activation foil is calculated by:

$$
a(t)=\lambda_{n} m_{o} \int_{0}^{\infty} \sigma_{m n}(E) \int_{0}^{t} \phi\left(E, t^{\prime}\right) d t^{\prime} d E
$$

where $\lambda_{n}$ is the decay constant for nucleus $\mathrm{n}, m_{o}$ is the $\mathrm{e}^{155}$ initial number of target atoms, $\sigma_{m n}$ is the cross section for the reaction producing the atom $\mathrm{n}$ and $\phi$ is the differential flux, which is a function of energy and time. The calculated activities are compared with the measured activities to obtain a weighting function. The resulting correction ${ }^{160}$ term is given by

$$
C_{j}^{[k]}=\sum_{i=1}^{n} w_{i, j}^{[k]} \ln R_{i}^{[k]} / \sum_{i=1}^{n} w_{i, j}^{[k]}
$$

where $R_{i}^{[k]}$ is the ratio of measured to calculated activity and $w_{i, j}^{[k]}$ is the activity weighting function:

$$
w_{i, j}^{[k]}=\frac{1}{2}\left(A_{i, j}^{[k]}+A_{i, j-1}^{[k]}\right) / A_{i}^{[k]} .
$$

The flux distribution is then adjusted:

$$
\phi_{j}^{[k+1]}=\phi_{j}^{[k]} \exp c_{j}^{[k]}
$$

These equations are applied iteratively until the solution converges to a specified standard deviation between calculated and measured activities or a change of less than $0.1 \%$ between the standard deviations of run iterations is achieved.

Modification of the SAND-II code was required to adjust fluxes up to $100 \mathrm{MeV}$, as the code has defined energy bins that extend only to $20 \mathrm{MeV}$. Historically, this procedure has been applied to reactors and critical assemblies where the neutron energies are well within the $20 \mathrm{MeV}$ maximum window allowed by the code. The energy binning was adjusted to accommodate these larger energies, now extending to $200 \mathrm{MeV}$ to allow for future adjustments of faster spallation neutron fluxes. A new cross section library was also required as the energy bins of the cross section library must match those of the adjustment code. Activation reactions were selected from those measured in the experiment based on the availability of experimental data to verify the theoretical calculation of the cross section.

Appropriate activation foil reactions were selected based on the energy range of the peak of the differential cross section and the quality of available nuclear data for the reaction. Activities for each foil were calculated in the code from the cross section library and the input flux. The flux was adjusted based on this calculation, leading to an inherent assumption that the nuclear data provided to the code is correct. It is therefore important to consider the selection of included nuclear data and simulation carefully. Cross section libraries compiled by P. Griffin from evaluated data are available for energies below $20 \mathrm{MeV}$ [5], but similar work has not been done for higher energies. There is also very little experimental data for neutron-induced reactions at energies above $40 \mathrm{MeV}$. For this reason, the TALYS-based Evaluated Nuclear Data Libraries (TENDL) is often used to estimate reaction rates for these higher energies. In order to extend the energy region over which the SAND-II code is able to adjust the neutron spectrum, a new cross section library was created using TALYS/TENDL results consisting only of reactions with some experimental data to verify the simulation.

Cross section files were obtained from the TENDL-2014 library up to $60 \mathrm{MeV}$ 6]. Above $60 \mathrm{MeV}$, the cross sections were calculated by running the code TALYS with the available TENDL-2014 input parameters. The resultant differential cross sections for the selected reactions are shown in Fig 2 with all available EXFOR experimental data. For selected reactions, calculated cross sections agree with experimental data within error. Reactions with multiple channels to produce the final observed nucleus, such as ${ }^{27} \mathrm{Al}(\mathrm{n}, \mathrm{x}){ }^{24} \mathrm{Na}$, were considered as a sum of all contributing reaction channels. In the case of the formation of ${ }^{24} \mathrm{Na}$, only data for the $(\mathrm{n}, \alpha)$ channel is available in EXFOR. The higher energy structure seen in the TALYS output is the result of $(n, 2 n 2 p)$ and $\left(n, n^{3} \mathrm{He}\right)$ reactions.

The TENDL/TALYS-2014 cross sections were parsed to ENDF format prior to processing with NJOY [7] and 

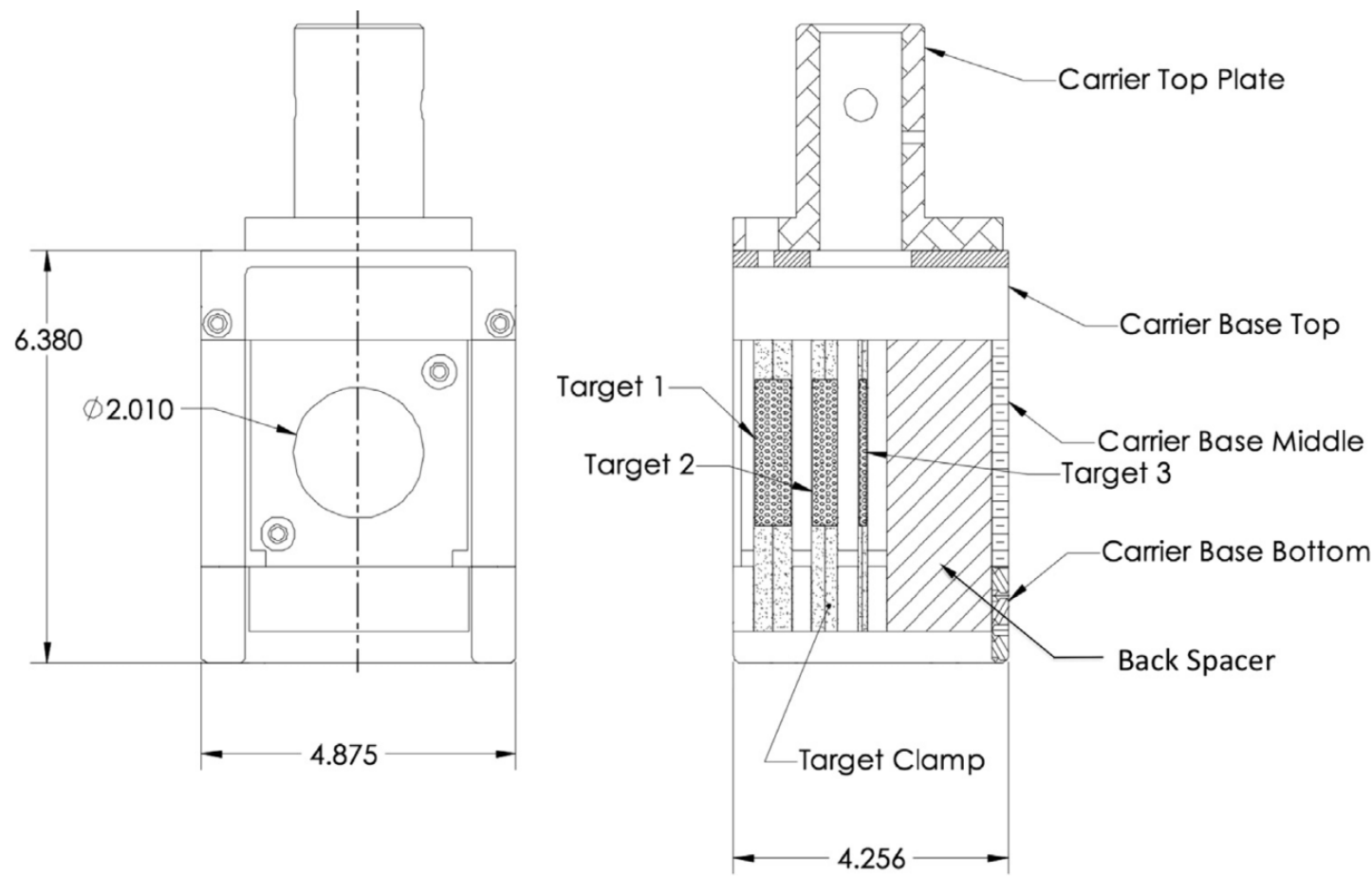

Figure 1: Schematic of the IPF target stack from the front (left) and side (right). All dimensions are in inches.

the final creation of a new cross section library for SANDProcessing through NJOY was done parameters used to create the snlrml-ii library [5], compiled by P. Griffin, with an adjusted energy binning scheme to match the higher energy modified SAND-II bins. The205 NJOY processing controls interpolation of the cross secThis output was then post-processed to match the format required for the SAND-II routine CSTAPE, which handles cross sections. No covariance data were supplied as210 currently distributed version of the TALYS code does not

\subsection{Activation product yield}

Experimental yields at end of irradiation determined from fitting decay curves are tabulated in Table 1. Only the reactions used in the spectral adjustment are shown.215 As the production of the radioisotope ${ }^{67} \mathrm{Cu}$ is specifically mentioned as a candidate for production in the secondary neutron field, it is worth mentioning that its yield was measured to be $7.608 \cdot 10^{-5} \mu \mathrm{Ci} / \mathrm{mg} / \mu \mathrm{A}$ from a natural $\mathrm{Zn}$ foil. For additional yield data, see Ref. 1].

\section{Results}

The activities calculated from the adjusted flux and input cross sections show good agreement with the measured ${ }^{225}$ activities for the selected reactions. As indicated in Fig. 3. the neutron spectrum was adjusted from $8 \mathrm{MeV}-60 \mathrm{MeV}$, based on the convolution of differential cross section and differential flux. The horizontal bands in this figure indicate the energy region over which $90 \%$ of the activity for each calculated reaction was produced, with $5 \%$ above and $5 \%$ below the band, and the vertical position of each band gives the deviation between the experimentally measured activity and the calculated activity. Energy regions with no contributing reactions were not adjusted in the code. The input MCNPX neutron distribution was normalized to the total calculated fluence above $1 \mathrm{MeV}$ obtained from SAND-II.

\section{Discussion}

The adjustment procedure revealed only minor changes in the shape of the neutron flux distribution from the MCNPX calculation, with the most pronounced at roughly $10 \mathrm{MeV}$. This is close to the lower energy bound for spectral adjustment, so it is difficult to ascertain the details of the deviation at low energy.

A comparison of the calculated activities from the SANDII-adjusted flux and a number of other calculations is shown in Fig. 4. All model calculations assume the MCNPX neutron flux. The model calculations Bertini [9] and CEM03.02 [10] were done using MCNPX event generators. Bertini is the default MCNPX option, in which Bertini IntraNuclear Cascade (INC) follows the Multistage Preequilibrium Model (MPM) 11] and evaporation described by Desner's EVAP code and the RAL fission model 12. The improved Cascade-Exciton Model (CEM) is implemented in CEM03.02. 

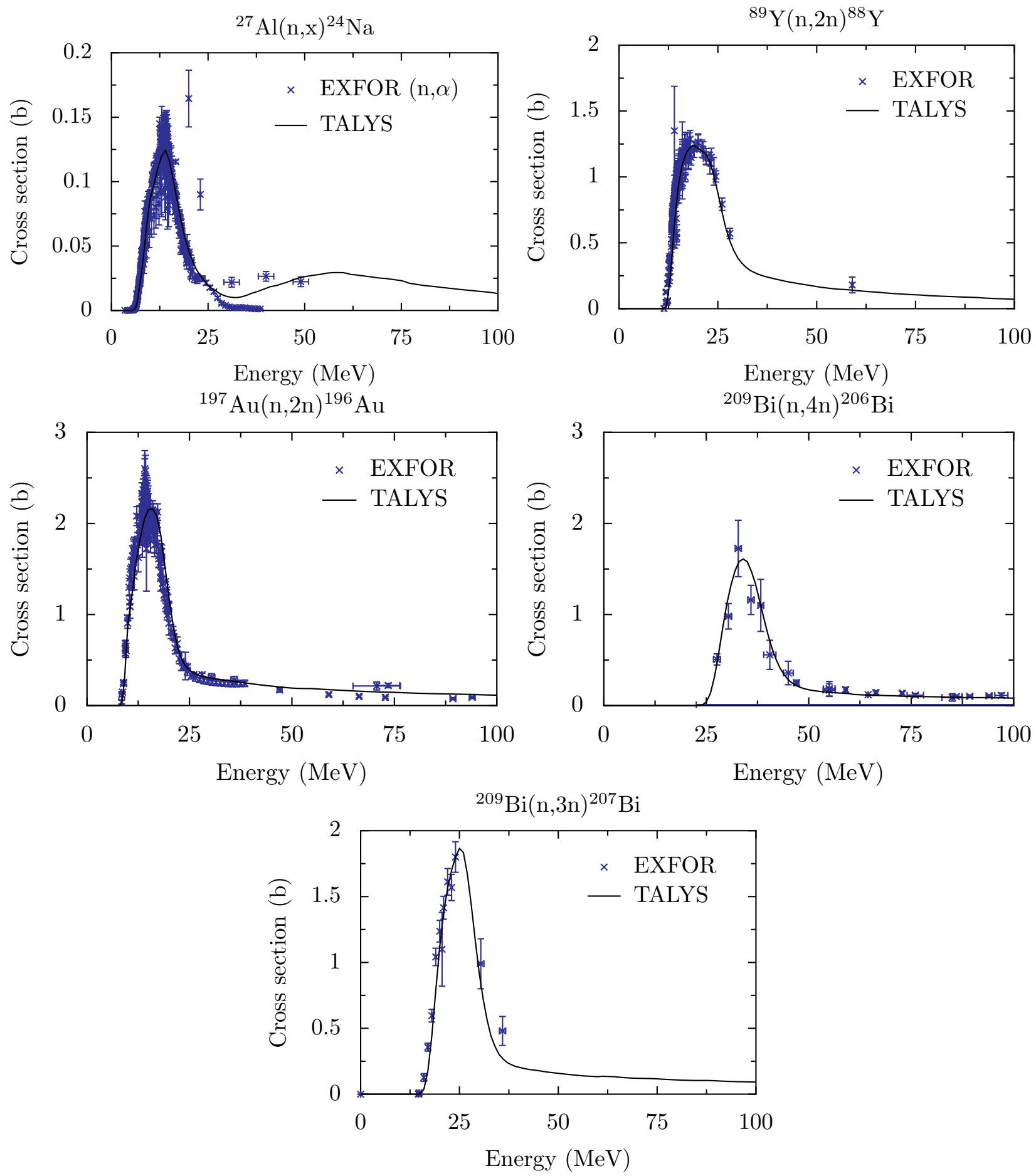

Figure 2: Comparison of TALYS/TENDL-2014 simulated cross sections with available EXFOR data for the foil reactions selected to be used in the adjustment procedure. 


\begin{tabular}{lccc}
\hline Reaction & $\mathrm{t}_{1 / 2}(\mathrm{~d})$ & Experimental yield $(\mu \mathrm{Ci})$ & Uncertainty $(\mu \mathrm{Ci})$ \\
\hline${ }^{27} \mathrm{Al}(\mathrm{n}, \alpha)^{24} \mathrm{Na}$ & 0.625 & 36.6 & 1.1 \\
${ }^{89} \mathrm{Y}(\mathrm{n}, 2 \mathrm{n})^{88} \mathrm{Y}$ & 106.6 & 0.47 & 0.03 \\
${ }^{197} \mathrm{Au}(\mathrm{n}, 2 \mathrm{n}){ }^{196} \mathrm{Au}$ & 6.2 & 24.7 & 0.8 \\
${ }^{209} \mathrm{Bi}(\mathrm{n}, 3 \mathrm{n})^{207} \mathrm{Bi}$ & 11523.6 & 0.017 & 0.001 \\
${ }^{209} \mathrm{Bi}(\mathrm{n}, 4 \mathrm{n})^{206} \mathrm{Bi}$ & 6.2 & 20 & 1 \\
\hline
\end{tabular}

Table 1: Tabulation of end of irradiation yields for reactions used in adjustment from [1].

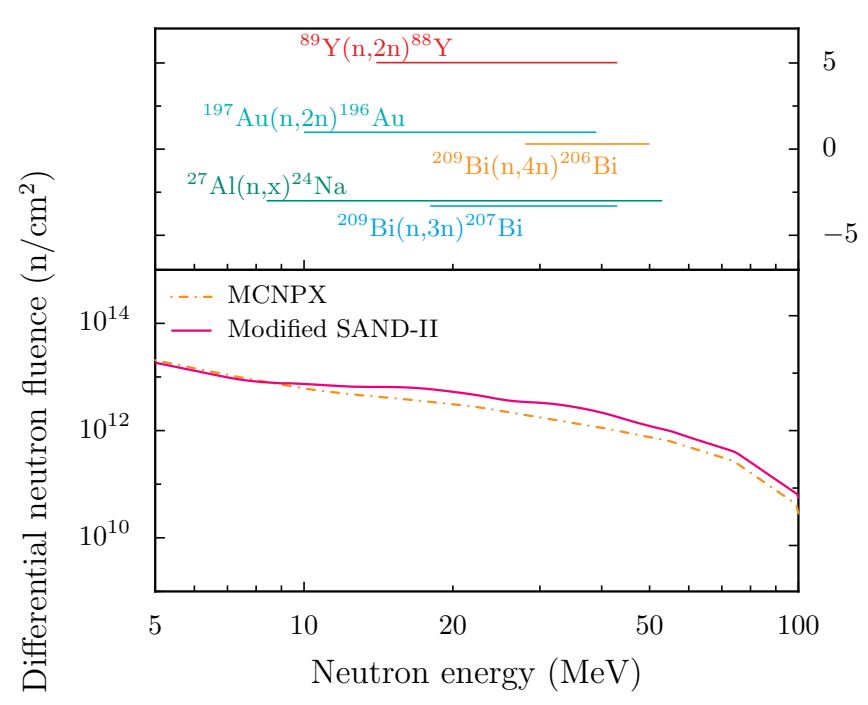

Figure 3: Summary of output from the SAND adjustment procedure. The length of each line in the top foil contribution plot gives the energy region over which that foil contributes to the adjustment procedure. The vertical position of each line gives the deviation between the experimentally measured activity and the activity calculated using the final SAND-modified flux with the cross sections shown in Fig. 2

A direct comparison of the activities resulting from the adjustment procedure with those from TENDL-2011 shows the influence of the spectrum adjustment. In all cases, the adjusted flux leads to better agreement with ${ }^{255}$ measured activities than the MCNPX flux. The adjusted differential neutron flux obtained from the radioactivation foil analysis provides critical, experimentally-verified information about the feasibility of symbiotic use of the spallation neutron flux for radionuclide production.

\section{Conclusion}

Radionuclide production data was used in a statistical adjustment procedure to experimentally determine the ${ }_{265}$ secondary neutron flux created at IPF. The resulting spectrum shows small changes from the MCNPX simulation of the experimental configuration. The good agreement of the calculated activities for each reaction foil with the ${ }_{270}$ measured activities indicates that the neutron flux is constrained and understood. With this knowledge, the secondary neutron flux at IPF can be used for radionuclide

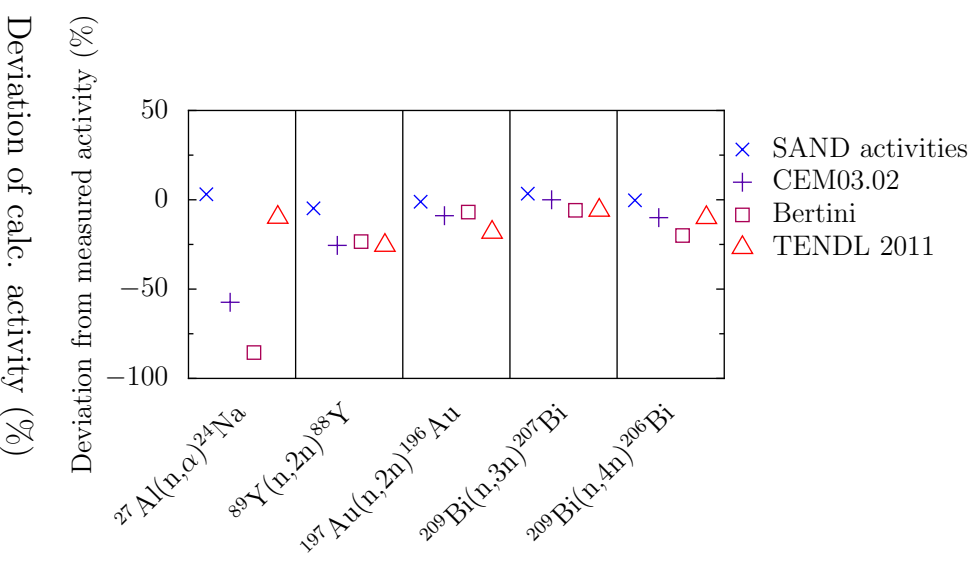

Figure 4: Percent difference between the experimentally measured activities and those calculated from models for the reactions used in the adjustment procedure.

production without modification of the standard proton target stack.

\section{Acknowledgments}

This study was carried out under the auspices of the National Nuclear Security Administration of the U.S. Department of Energy at Los Alamos National Laboratory under Contract No. DE-AC52-06NA253996 with partial funding by the U.S. DOE Office of Science via funding from the Isotope Development and Production for Research and Applications subprogram in the Office of Nuclear Physics.

\section{References}

[1] J. W. Engle, M. R. James, S. G. Mashnik, C. T. K. Iv, L. E. Wolfsberg, D. A. Reass, M. A. Connors, H. T. Bach, M. E. Fassbender, K. D. John, E. R. Birnbaum, F. M. Nortier, MCNPX characterization of the secondary neutron flux at the Los Alamos Isotope Production Facility Nuclear Inst. and Methods in Physics Research, A 754 (2014) 71-82. doi:10.1016/j.nima. 2014.03 .049

URL http://dx.doi.org/10.1016/j.nima.2014.03.049

[2] J. W. Engle, S. G. Mashnik, H. Bach, A. Couture, K. Jackman, R. Gritzo, B. D. Ballard, M. Fassbender, D. M. Smith, L. J. Bitteker, J. L. Ullmann, M. S. Gulley, C. Pillai, K. D. John, E. R. Birnbaum, F. M. Nortier, Cross sections from $800 \mathrm{MeV}$ proton irradiation of terbium. Nuclear Physics A 893 (2012) 87100. doi:10.1016/j.nuclphysa.2012.08.001 URL http://dx.doi.org/10.1016/j.nuclphysa.2012.08.001 
[3] J. W. Engle, S. G. Mashnik, J. W. Weidner, L. E. Wolfsberg, M. E. Fassbender, K. Jackman, A. Couture, L. J. Bitteker, J. L. Ullmann, M. S. Gulley, C. Pillai, K. D. John, E. R. Birnbaum, F. M. Nortier, Cross sections from proton irradiation of thorium at $800 \mathrm{MeV}$, Physical Review C - Nuclear Physics 88 (1) (2013) 1-8. doi:10.1103/PhysRevC.88.014604

[4] P. J. Griffin, SAND-II Manual, SAND93-3957, Sandia National Laboratories.

[5] P. J. Grif, C. D. Peters, D. W. Vehar, Recommended Neutron Dosimetry Cross Sections for the Characterization of Neutron Fields, IEEE Transactions on Nuclear Science 59 (4) (2012) $1167-1174$.

[6] A. J. Koning, D. Rochman, Modern Nuclear Data Evaluation with the TALYS Code System Nuclear Data Sheets 113 (12) (2012) 2927-2934. doi:10.1016/j.nds.2012.11.002 URL http://dx.doi.org/10.1016/j.nds.2012.11.002

290 [7] R. E. MacFarlane, a. C. Kahler, Methods for Processing ENDF/B-VII with NJOY, Nuclear Data Sheets 111 (6972) (2010) 2739-2890. doi:10.1016/j.nds.2010.11.001

[8] D. Rochman, Private communication (July 2015).

[9] H. Bertini, Physical Review 188 (1969) 1711.

[10] S. Mashnik, K. Gudima, R. Prael, A. Sierk, M. Baznat, N. Mokhov, Cem03.03 and laqgsm03.03 event generators for the mcnp6, mcnpx, and mars15 transport codes, la-ur-08-2931, invited Lectures Presented at the Joint ICTP-IAEA Advanced Workshop on Model Codes Spallation Reaction, February 4-8, 2008, ICTP, Los Alamos, Trieste, Italy, 2008 p. 51.

[11] R. Prael, M. Bozoian, Adaptation of the multistage preequilibrium model for the monte carlo method, LA-UR-88-3238.

[12] F. Atchison, Spallation and fission in heavy meatl nuclei under medium energy proton bombardment, Proceedings of the Meeting on Targets for Neutron Beam Spallalation Sources, Julich, Germany, 1980 pp. 17-46. 